\title{
Latency Hiding in Dynamic Partitioning and Load Balancing of Grid Computing Applications
}

\author{
Sajal K. Das ${ }^{1}$, Daniel J. Harvey ${ }^{1}$, and Rupak Biswas ${ }^{2}$ \\ ${ }^{1}$ Department of Computer Science \& Engineering \\ The University of Texas at Arlington \\ Arlington, TX 76019-0015, USA \\ E-mail:\{das, harvey\}@cse.uta.edu \\ ${ }^{2}$ NASA Ames Research Center \\ Mail Stop T27A-1 \\ Moffett Field, CA 94035-1000, USA \\ E-mail: rbiswas@nas.nasa.gov
}

\begin{abstract}
The Information Power Grid (IPG) concept developed by NASA is aimed to provide a metacomputing platform for large-scale distributed computations, by hiding the intricacies of a highly heterogeneous environment and yet maintaining adequate security. In this paper, we propose a latency-tolerant partitioning scheme that dynamically balances processor workloads on the IPG, and minimizes data movement and runtime communication. By simulating an unsteady adaptive mesh application on a wide area network, we study the performance of our load balancer under Globus environment. The number of IPG nodes, the number of processors per node, and the interconnect speeds are parameterized to derive conditions under which the IPG would be suitable for parallel distributed processing of such applications. Experimental results demonstrate that effective solutions are achieved when the nodes of the IPG are connected by a high-speed asynchronous interconnection network.
\end{abstract}

\section{INTRODUCTION}

The Information Power Grid (IPG) infrastructure has been developed by NASA and other collaborative partners to harness the power of geographically distributed resources (computers, databases, and human expertise) in order to solve large-scale computational problems. Applications that would benefit from such an infrastructure include:

- Desktop coupling to remote supercomputers to provide access to large databases and high-end graphics facilities [9].

- User access to sophisticated instruments through remote supercomputer connections utilizing virtual reality techniques [8]. - Remote interactions with supercomputer simulations [10], [11].

Several attempts have been recently made to develop what are called computational grid capabilities and/or implementations. (See [14] for a comprehensive survey of current technology.) For example, the Condor system [19] is developed to manage research studies at workstations around the world. However, it did not adequately deal with the security issues involved. Other grid-based systems include Nimrod [1], NetSolve [4], NEOS [5], Legion [15], and CAVERN [18]. The Globus Metacomputing Infrastructure Toolkit [13] successfully provides a portable virtual machine environment. It supports

This work was supported by NASA Ames Research Center under Cooperative Agreement Number NCC 2-5393. mechanisms for sharing remote resources, provides adequate security, and allows MPI-based message passing. Due to its portable and modular nature, Globus has been chosen by NASA as the middleware to implement the IPG.

So far, limited studies have been performed to determine the viability of parallel distributed computing on the IPG. In [2], latency tolerance and load balancing modifications were implemented for a CFD application to compensate for slower communication speed. Results showed that the application ran faster under Globus on two IPG nodes of four processors each than on a single tightly-coupled machine of eight processors. However, this result is clouded in that asynchronous message passing was supported over the high-speed link but not within the single platform. With a goal to make more informative conclusions, in this paper we simulate an unsteady adaptive mesh application on a wide area network. The number of IPG nodes, the number of processors per node, and the interconnect speeds are parameterized to derive conditions under which the IPG would be suitable for parallel distributed processing of such applications.

Earlier, we proposed two different load balancing approaches with an unsteady adaptive mesh as the test case application. The first approach, called PLUM [21], is an architectureindependent framework which globally partitions the computational mesh after each adaptation and determines whether rebalancing the load would lead to reduced total execution time. If an improvement in the load balance can be achieved, PLUM utilizes an effective re-mapping algorithm to minimize the required data movement. Application processing is temporarily suspended during the partitioning and data re-mapping operations. Utilization of a parallel graph partitioner like ParMeTiS [17] gives effective results.

The second approach, called Symmetric Broadcast Networks (SBN) [7], gives a general-purpose topology-independent solution to dynamic load balancing. A salient feature of this approach is that it balances processor workloads while the application is running. Therefore, it is able to hide the high data migration overhead, albeit at the cost of increased interprocessor com- 
munication. Results reported in [3] indicate that both PLUM and SBN approaches have their relative merits, and that they achieve excellent load balance with minimal extra overhead.

Let us summarize the contributions of this paper. We propose a novel partitioner, called MinEX, that optimizes the two important steps of PLUM (balancing and re-mapping) as part of the partitioning process. Instead of attempting to only balance the load like most other partitioners, the objective of MinEX is to minimize the total runtime of the application. This approach counters the possibility that perfectly balanced loads can still incur excessive communication and redistribution costs while the application is being processed. MinEX is also used to experiment with the latency tolerant techniques on the IPG. Our experimental results show that MinEX reduces the number of elements migrated by PLUM, and also lowers the percentage of edges cut by SBN. For example, for 32 partitions with our test case, PLUM showed an edge cut of $10.9 \%$ and redistributed 63,270 mesh elements. The corresponding values for the SBNbased approach were $36.5 \%$ and 19,446 . In contrast, the MinEX partitioner values were $20.9 \%$ and 30,548 respectively. Thus MinEX attempts to optimize both communication and remapping costs, and hence is found to be an effective approach to latency hiding in dynamic load balancing for grid computing.

This paper is organized as follows. Section 2 introduces the computational application to be tested and determines its scalability. Section 3 describes the new MinEX partitioner. Section 4 describes the experimental study, analyzes the obtained results and draws conclusions as to the use of the IPG for this and similar applications. Section 5 concludes the paper.

\section{Test CASE Scenario}

Many computational problems are often modeled as an unstructured mesh of vertices and edges. To capture evolving features, the mesh topology is also frequently adapted. For an efficient parallel implementation, this leads to dynamic load balancing in the sense that mesh objects will have to be reassigned after each adaptation phase to re-balance the workload among processors. Traditional solutions to this application invoke a partitioner after each adaptation step to accomplish the rebalancing followed by the data set reassignment among processors. MinEX combines the partitioning and data set reassignment steps. For efficient parallel implementations, it is critical to minimize the overhead associated with re-mapping data sets, and to reduce the communication between processors at the next solution step. These goals are particularly important in the IPG context where communication bandwidth between nodes are likely to be much smaller than those within a single multiprocessor machine.

The computational mesh considered for our experiments in this paper simulates an unsteady environment with a strongly time-dependent adapted region. As depicted in Fig. 1, a shock wave is propagated through an initial grid to produce the desired effect. The computational mesh is processed through nine adaptations by moving a cylindrical volume across the domain with constant velocity. Grid elements within the cylindrical volume are refined while previously-refined elements are coarsened in its wake. During the processing, the size of the mesh increases from 50,000 elements to $1,833,730$ elements.

To realistically simulate the overhead associated with an

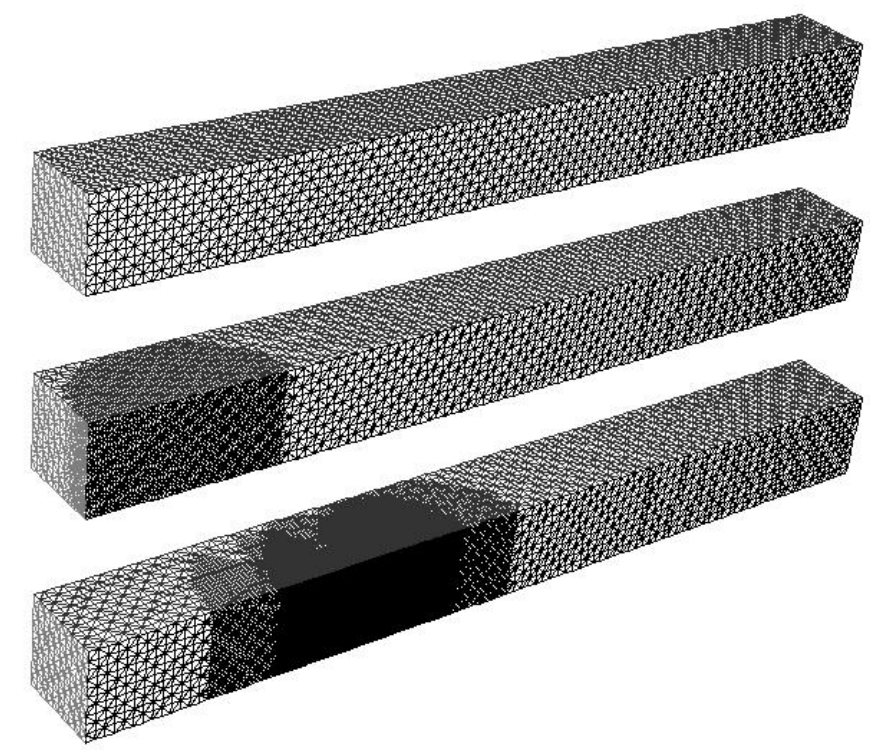

Fig. 1. Initial and adapted meshes (after levels 1 and 5) for the simulated unsteady experiment.

adaptive mesh computation, two weights are associated with each mesh vertex and one weight with each mesh edge. These weights respectively reflect the number of time units required for computation, data remapping, and communication cost. The total time required to process the vertices assigned to a processor $p$ must take into account all these three metrics as defined below.

Processing Weight, $W g t^{v}$, is the computational cost to process a vertex $v$.

Redistribution Cost, Remap $v$, is the overhead to copy from processor $p$ to another processor the data set associated with $v$. This cost incurred at $p$ includes operations like data packing and initiating transmission. The redistribution cost incurred by the processor receiving $v$ is the sum of the communication cost and the operations of unpacking and merging the data into existing data structures. Clearly, if the data set for vertex $v$ is already assigned to processor $p$, no redistribution cost is incurred, i.e. $\operatorname{Remap}_{p}^{v}=0$.

Communication Cost, $\operatorname{Comm}_{p}^{v}$, is the cost to interact with all vertices adjacent to $v$ but whose data sets are not local to processor $p$. Define $\operatorname{Comm}_{p}^{(v, w)}$ to be an edge weight that represents the cost to interact with a single vertex, $w$ adjacent to $v$. Note that if the data set associated with $w$ is local to processor $p$, $C o m m_{p}^{(v, w)}=0$. If the data sets of all the vertices adjacent to $v$ are also assigned to $p$, the communication cost, $\operatorname{Comm}_{p}^{v}$, is 0 .

We also use six additional metrics which are defined as follows.

Weighted Queue Length, QWgt $(p)$, is the total cost to process the vertices assigned to $p$. It is defined as:

$$
\operatorname{QWgt}(p)=\sum_{v \text { assigned to } p}\left(W g t^{v}+\operatorname{Comm}_{p}^{v}+\operatorname{Remap}_{p}^{v}\right)
$$

Total System Load, QWgt TOT, is the sum of QWgt $(p)$ over all processors. This metric will be used in Section III-B to decide whether it is appropriate to reassign a vertex from one processor to another. 
Heaviest Load, MaxQWgt, is the maximum value of QWgt $(p)$ over all processors, and indicates the total time required to process the application.

Lightest Load, MinQWgt, is the minimum value of $Q W g t(p)$ over all processors, and indicates the workload of the most lightly-loaded processor.

Average Load, AvgQWgt, is $Q W g t$ TOT/ $P$, where $P$ is the total number of processors.

Load Imbalance Factor, LoadImb, represents the quality of the partitioning and is defined as MaxQWgt / AvgQWgt.

Table I shows the scalability of our test case application where the number of processors, $P$, is varied from 2 to 2048 . The data was obtained by simulating the application (details presented in Section IV). Each column reflects non-dimensionalized MaxQWgt values in thousands. The first row of the table assumes that maximum latency tolerance is achieved, while the second row assumes that no latency tolerance is achieved. By maximum latency tolerance, we mean the ability to utilize all available processors to overlap communication and redistribution costs. Further explanations are provided in Section III. Table I shows that this application can scale to over 128 processors with linear speedup, and therefore is a good potential for an IPG implementation.

\section{MineX: A New Partitioner}

Previous studies with the above mesh application under the PLUM framework utilized a variety of general partitioners such as ParMeTiS [17], UAMeTiS [22], DAMeTiS [22], JostleMS [23], and Jostle-MD [23]. Note that UAMeTiS, DAMeTiS, and Jostle-MD are diffusive schemes designed to modify existing partitions to produce a processor allocation; whereas PMeTiS and Jostle-MS are global partitioners which makes no assumptions about the original mesh distribution. Although all these partitioners achieve good load balance while minimizing communication overhead, they fail to consider the cost of moving data between processors. A unique feature of PLUM is to address this drawback through the use of an efficient heuristic procedure for redistributing data to assigned processors.

In the following, we design, implement, and analyze a novel partitioner, called MinEX, that optimizes computational, communication, and data remapping costs. We also redefine the partitioning goal from producing balanced loads to minimizing MaxQWgt. No direct comparisons with other existing partitioners mentioned above are possible because MinEX also considers the data redistribution cost while partitioning the computational mesh.

\section{A. Design Principles}

MinEX can be classified as a diffusive multilevel partitioner. Diffusive algorithms [6] utilize an existing partition as a starting point instead of partitioning from scratch. The multi-level approach, originally introduced in [16], partitions the graph in three steps - contraction, partitioning, and refinement - each of which is described below.

Similar to other multilevel partitioners, the first step in MinEX is to contract the mesh to a reasonable size. What is different, however, is the contraction procedure. Instead of repeatedly contracting the mesh in halves as is common with other multilevel partitioners, MinEX sequentially contracts one vertex at a time. The advantage of this approach is that a decision can be made each time a vertex is later refined as to whether it should be assigned to another processor. This makes the algorithm more flexible since the graph does not have to be doubled in size before this decision could be made. If $|V|$ is the number of vertices in the mesh, contraction requires $O(|V|)$ steps. The total asymptotic complexity is no larger than that of contracting the mesh sequentially in halves.

Once the mesh is sufficiently contracted, the remaining vertices are reassigned according to the partitioning criteria described in detail in Section III-B below.

The mesh is expanded back to its original size through a refinement process. As each vertex is refined, a decision is made as to whether or not it should be reassigned. This decision employs the same partitioning criteria followed by the partitioning algorithm in the above step. Each coarse vertex reassignment in effect reassigns all of the computational vertices that the coarse vertex represents.

\section{B. Partitioning Criteria}

The criteria for deciding whether a vertex should be reassigned from one processor to another, is based on two metrics: Gain and MinVar.

Gain represents the change in QWgtTOT that would result from a proposed vertex move. A negative Gain value would indicate that less total processing is required after such a vertex reassignment. The partitioning algorithm favors vertex moves with negative or small Gain values that reduce or minimize overall system load.

MinVar is computed using the workload (i.e. $\mathrm{QWgt}(p)$ ) for each processor $p$ and the smallest load of any processor (MinQWgt) in accordance with the following formula:

$$
\operatorname{MinVar}=\sum_{p}(\operatorname{QWgt}(p)-\operatorname{MinQWgt})^{2} .
$$

In other words, MinVar computes the variance of processor workloads from that of the most lightly-loaded processor. The objective is to initiate vertex moves that lower this value. Since processors with large $Q W g t(p)$ values will have large MinVar components, this criteria will tend to move vertices away from processors that have high runtime requirements. $\Delta$ MinVar is the change in the MinVar value after moving a vertex from one processor to another. A negative value indicates that the MinVar metric has been reduced.

Let us now describe how the partitioning decisions are made. For each vertex, $v$, consider all edges to adjacent vertices that are assigned to other processors. Compute the Gain and MinVar values that would result from moving $v$ to each of the adjacent processors. The vertex move that is executed is the one with the smallest value of Gain as long as $\Delta$ MinVar $<0$ and -Gain/DMinVar < ThroTtle. To increase efficiency, the program utilizes a minimum heap with vertex pointers to heap locations to quickly find the best move and directly delete heap entries without searching.

Conceptually, ThrotTle acts as a gateway that limits increases in Gain based upon how much of an improvement 
in MinVar can be achieved. Table II shows how varying ThroTTle values affects the expected application runtime (MaxQWgt) and load balance quality (LoadImb). The MaxQWgt entries are non-dimensionalized values in thousands. These results were obtained by running the experiments described in Section IV. Table II assumes a network of 32 homogeneous processors distributed over one to eight IPG nodes (clusters). The inter-cluster interconnect speed is assumed to be a third of the intra-cluster speed. Results show that a ThroT$\mathrm{Tle}$ value of 64 produces the lowest overall MaxQWgt, and that larger ThroTTle values improve LoadImb. Experiments with other network sizes using this same mesh have shown that ThroTtle generally converges at values between $P$ and $2 P$. Note also that for large values of ThroTTle, better LoadImb does not necessarily imply lower MaxQWgt.

\section{Latency Tolerance}

The following steps illustrate how communication and data redistribution can be reduced or eliminated from a given processor $p$.

Step 1: Initiate send of all data sets to be redistributed.

Step 2: For each edge $(v, w)$, where the data set for vertex $v$ is local to processor $p$ and the data set for vertex $w$ is local to another processor $q$, initiate send of communication data. The metric $\operatorname{Comm}_{p}^{(v, w)}$ represents the cost of this communication.

Step 3: Process vertices that are not waiting for incoming transmissions.

Step 4: Receive and unpack any re-mapped data sets destined for this processor.

Step 5: Receive and unpack communication data destined for this processor.

Step 6: Repeat Steps 2 through 5 until all vertices are processed.

The above steps implement a strategy where processors distribute data sets and communication data as early as possible. The processing of internal vertices can then take place while waiting for expected incoming messages. As data sets and communication data are received, additional communications can be initiated and vertices processed. The most optimistic expectation of this strategy is that the processing activity can entirely hide the data set redistribution cost and communication latency. At the other extreme, the most pessimistic view is that no latency tolerance is achieved. Experiments simulating both views to analyze the effect of latency tolerance on our test application are described in Section IV.

\section{Data Structures}

The following data structures are used by the MinEX partitioner to perform its multilevel algorithm:

- Mesh: The adaptive mesh has the format

$\left\{|V|,|E|, \mathrm{vTot},{ }^{\mathrm{VM}} \mathrm{VaP},{ }^{\mathrm{V}} \mathrm{VList}\right.$, *EList $\}$ where

- $|V|$ is the number of active vertices in the mesh,

- $|E|$ is the number of edges in the mesh,

- vTot is the total number of vertices (including merged vertices),

_ $\star$ VMaP is a pointer to the list of active vertices,

_ $\star$ VList is a pointer to the complete list of vertices,

- ${ }^{\star}$ EList is a pointer to the list of edges.
- VmaP: A list of active vertices. None of these vertices have been compressed through multilevel partitioning.

- VList: A complete list of vertices. Each vertex, $v$, is defined by a VList record as $\left\{W g t, \operatorname{Remap}_{p},|e|, * e\right.$, merge,

lookup, *vmap, *heap, border $\}$ where

- Wgt is the computational cost to process $v$.

- Remap is the redistribution cost to copy the data set associated with $v$ to another processor from $p$.

- $|e|$ is the number of adjacent edges associated with $v$.

- *e is a pointer to the first edge, $(v, w 1)$ in the EList structure (defined below) associated with $v$. Subsequent edges, $(v, w 2),(v, w 3), \cdots$ are stored in contiguous EList entries.

- merge is the vertex that was merged with $v$ during a contraction operation. It is set to -1 if no merge took place.

- lookup is the active vertex that contains $v$ after a series of contraction operations. It is also set to -1 if no merges took place.

$-* v m a p$ is a pointer to the position of $v$ in the active vertex table.

- *heap is the pointer to an entry in the heap that relates to vertex, $v$, and represents a potential reassignment of $v$. As mentioned in Section III-B, this pointer allows heap entries to be removed without searching.

- border is a boolean flag indicating whether $v$ is adjacent to vertices assigned to other processors.

- EList: A list of edges in the mesh. Each edge record is defined as $\left\{w i, \operatorname{Comm}_{(\mathrm{v}, w i)}\right\}$ where $(v, w i)$ is an edge and $\operatorname{Comm}_{(\mathrm{v}, \mathrm{wi})}$ is the communication weight associated with this edge. Vertex $v$ has an entry in VList and edges are located using the $* e$ pointer.

- Heap: The heap of potential vertex reassignments. Each heap record is defined as $\{\mathrm{Gain}, \Delta \mathrm{MinVar}, v, p\}$ which specifies the Gain and $\Delta$ MinVar that would result from reassigning vertex $v$ to processor $p$. The min-heap is keyed by the Gain value.

- Stack: The stack of compressed vertex pairs, $\left(v_{1}, v_{2}\right)$. These vertices are refined in reverse order from the order that they were compressed. This graph contraction technique is described below.

\section{E. Graph Contraction}

The partitioner selects sets of randomly chosen pairs of vertices that are assigned to the same processor $p$. From this set, the vertex pair, $(v, w)$, that has the largest $\operatorname{Comm}_{(v, w)} /\left(\operatorname{Remap}_{p}^{v}+\right.$ Remap $\left.p_{p}^{w}\right)$ value is merged. This formula attempts to find edges with large communication costs while minimizing the potential cost of data set redistribution. The motivation behind this strategy is to arrive at a contracted mesh with a small edge cut and a small data distribution cost.

To contract a pair of vertices, a merged vertex record, $M$, is created and the edge $(v, w)$ is collapsed. The edge records corresponding to $M$ are created utilizing the edge lists of vertices $v$ and $w$. VMap is adjusted to contain the newly created vertex and to remove $v$ and $w ;|V|$ is decremented and vTot is incremented; $|E|$ is increased by the number of edges created for $M$; and the pair $(v, w)$ is pushed onto Stack.

This contraction procedure is implemented using a set union/find algorithm so that edges of existing vertices can re- 
main unchanged. For example, if an existing vertex is adjacent to $v$, accesses to its EList record will check whether $v$ has been merged. If it has, lookup will be accessed to quickly find the appropriate merged vertex. If lookup is not current (lookup > vTot), the union/find algorithm will search the chain of vertices beginning with merge in order to update the lookup value so subsequent lookups can be done efficiently. Pseudo code describing the union/find procedure is given in Fig. 2.

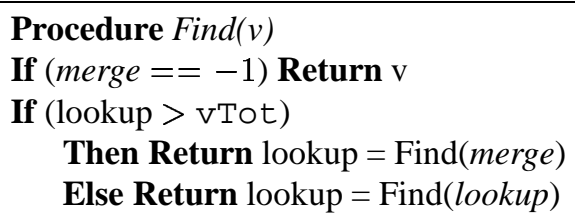

Fig. 2. Pseudo code for the union/find algorithm

\section{F. Partitioning the Contracted Graph}

Once the graph contraction process is complete, the partitioning can be performed. Because the number of vertices is greatly reduced, the MinEX partitioning algorithm can execute efficiently. The algorithm considers every remaining vertex of the mesh to find potential reassignments that will reduce Gain and MinVar as described in Section III-B. All potential vertex reassignments are added to the min-heap. Actual reassignments are executed in heap order. As a reassignment is executed, the heap is adjusted to reflect the new partition status.

\section{G. Refinement}

The graph is restored to its original size by expanding pairs of vertices in an order reversed from which they were merged. The Stack data structure controls the order. As pairs of vertices, $(v, w)$, are refined, merged edges and vertices are deallocated. The merge and lookup vertex numbers are also adjusted in the vertex table. The VMap table is adjusted to delete the merged vertex, $M$, and to add $v$ and $w .|V|$ is incremented and vTot is decremented; $|E|$ is decreased by the number of edges created for $M$. After each refinement, a decision is made as to whether a partition can be improved by reassigning $v$ or $w$. When reassignments are made, adjacent border vertices are also considered.

\section{Performance Study}

The MinEX partitioner was executed with actual application data to simulate an adaptive mesh computation for a variety of system configurations. Individual runs model networks with a particular number of processors $(P)$, number of IPG nodes/clusters $(C)$, ThroTTle values, and interconnect speeds $(I)$. In our experiments, $P$ was varied from 2 to 2048; $C$ was varied from 1 to 8 ; ThroTTle was varied to find the optimal value for minimizing runtime; and $I$ was varied to simulate both high-speed cluster interconnects and low-speed wide area network connections.

Based on performance studies reported in [12], [20], typical communication latency and bandwidth slowdown from integrated clusters to configurations with clusters connected through a high-speed interconnect are in the range of 3 to 100 . Wide area network connections are 1,000 to 10,000 times slower than the internal intra-connects of a single cluster. In our experiments, we have assumed that the intra-cluster communication speed is normalized to a value of 1 . Simulations of inter-cluster communication assumed slowdown factors of $3,10,100$, and 1,000. To simplify the analysis, we have assumed that individual processors are homogeneous and divided as evenly as possible among the clusters.

\section{A. Experimental Results}

Table III(a) and III(b) show results of experimental runs analyzing the effect of varying numbers of clusters and interconnect speeds, assuming $P=32$ homogeneous processors. The interconnect speeds indicate the slowdown factor relative to the intra-cluster communication speed. To be consistent with results presented in Tables 1 and 2, runtime are shown as nondimensionalized values in thousands. Table III(a) charts the experimental results when no latency tolerance is achieved; and Table III(b) assumes maximum latency tolerance.

The following conclusions can be drawn from the experiments.

- As the interconnect speed is reduced, the slowdown experienced by utilizing additional clusters increases dramatically. For example, the runtime metric in Table III(a) is 4,102 when two clusters and an interconnect slowdown of 1000 is assumed. However, the runtime metric is 93,566 when eight clusters are assumed. Thus the ratio is $93,566 / 4,102 \approx 22.80$. If we consider the interconnect slowdown of 3 , the ratio between two clusters and eight clusters is $968 / 728 \approx 1.32$ which is a much smaller value. The same pattern holds true in Table III(b).

- For the mesh application considered, Globus over low-speed networks such as the Internet is not a viable approach assuming current technology. In fact, the interconnection speed must improve by at least an order of magnitude before this approach could be useful. Under current technology, applications would have to have little runtime communication and data-set re-mapping for low-speed wide area networks to be practical interconnects.

- We can compare the effectiveness of latency tolerant algorithms to algorithms without latency tolerance, by measuring runtimes of each approach as the number of clusters and interconnect speeds are varied. The runtime improvements using latency tolerance increase as the number of clusters increase. This can be verified by comparing the same rows from Table III(a) and Table III(b). For example, consider the row with 6 clusters. The improvement in runtime comparing latency tolerant algorithms to those with no latency tolerance are $915-345=570,1717-856=861,8521-5044=$ $3477,76169-52668=23501$ respectively for interconnect slowdowns $3,10,100,1000$. In contrast the row with 2 clusters indicate indicate improvement in latency tolerance runtime gains of $728-298=430,863-469=394,1228-763=$ $465,4102-3941=161$ for the same interconnect slowdowns. In general, the rows that correspond to more clusters show greater runtime improvement when employing latency tolerance. The same cannot be said when analyzing columns of the tables where interconnect slowdowns are varied. For example, the table columns with interconnect slowdowns of 100 clusters indicate improvements in runtime by utilizing latency tolerance 
of $473-287=186,1228-763=465,2783-2386=$ $397,3667-3297=370,5677-4369=1308,8521-5044=$ $3477,10958-5480=5478,11492-5721=5771$ respectively for clusters 1 to 8 . The columns with interconnect slowdonws of 10 show corresponding runtime improvements of $473-287=$ $186,863-469=394,1168-548=620,1361-680=$ $681,1649-768=881,1717-856=861,1915-893=$ $1022,2178-1048=1130$. In this case, a clear pattern cannot be established.

- Note that the table columns corresponding to interconnect slowdown of 1000 shows a slowdown when utilizing latency tolerance if the number of clusters is 4 . This likely can be attributed to not running the latency tolerance test with an optimal ThrotTle value.

- For our test application, Globus could be a viable approach if a high-speed interconnect (slowdown factor between 3 and 10) between clusters is utilized. The first column of Tables III(a) and III(b) comparing 1 and 8 clusters with an interconnect slowdown factor of 3, respectively, show a slowdown factor of 2.04 and 1.24. Similarly, the second column of the tables with an interconnect slowdown factor of 10 show slowdown factors of 4.60 and 3.65, respectively. These factors being smaller than the number of clusters indicate a speedup from when one cluster of $\frac{1}{8}$ the number of processors are used.

\section{CONCLUSIONS}

We presented a latency-tolerant partitioner, called MinEX, that not only balances processor workloads but also minimizes data movement and runtime communication, for adaptive mesh applications that are executed in a parallel distributed fashion on the IPG. Additional experiments that are planned will test MinEX performance in the context of different application classes and devise metrics to compare MinEX performance with other popular partitioning schemes. We also analyzed the conditions that are required for the IPG to be an effective tool for such distributed computations. Our results demonstrate that MinEX is a viable load balancer provided the nodes of the IPG are connected by a high-speed asynchronous interconnection network. We are currently implementing a parallel version of MinEX. An area of further research includes mathematical analysis of latency tolerance or slowdowns based on the interconnect speed, numbers of clusters employed, and the topology of the mesh.

\section{REFERENCES}

[1] D. Abramson, R. Sosic, J. Giddy, and R. Hall, "Nimrod: A tool for performing parameterized simulations using distributed workstations," 4th IEEE Symposium on High Performance Distributed Computing, 1995.

[2] S. Barnard, R. Biswas, S. Saini, R. Van der Wijngaart, M. Yarrow, and L. Zechtzer, "Large-scale distributed computational fluid dynamics on the Information Power Grid using Globus," 7th Symposium on the Frontiers of Massively Parallel Computation, 1999, 60-67.

[3] R. Biswas, S.K. Das, D.J. Harvey, and L. Oliker, "Parallel dynamic load balancing strategies for adaptive irregular applications," Applied Mathematical Modelling, to appear, 2000.

[4] H. Casanova and J. Dongarra, "NetSolve: A network server for solving computational science problems," Technical Report CS-95-313, University of Tennessee, 1995.

[5] J. Cryzyk, M. Meznier, and J. More, "The Network-Enabled Optimization System (NEOS) server,” Preprint MCS-P615-0996, Argonne National Laboratory, 1996.

[6] G. Cybenko, "Dynamic load balancing for distributed-memory multiprocessors," Journal of Parallel and Distributed Computing, 7 (1989) 279301

[7] S.K. Das, D. Harvey, and R. Biswas, "Parallel processing of adaptive meshes with load balancing," 27th International Conference on Parallel Processing, 1998, 502-509. (Extended version currently under revision for IEEE Transactons on Parallel and Distributed Systems.)

[8] T. Defanti, M.D. Brown, and R. Stevens, "Virtual reality over high-speed networks," IEEE Computer Graphics and Applications, 16 (1996) 42-43.

[9] T. Defanti, I. Foster, M. Papka, R. Stevens, and T. Kuhlfuss, "Overview of the I-Way wide area visual supercomputing," International Journal of Supercomputer Applications, 10 (1996) 123-130.

[10] D. Diachin, L. Freitag, D. Heath, J. Herzog, W. Michels, and P. Plassmann, "Remote engineering tools for the design of pollution control systems for commercial boilers," International Journal of Supercomputer Applications, 10 (1996) 208-218.

[11] T. Disz, M. Papka, M. Pellegrino, and R. Stevens, "Sharing visualization experiences among remote virtual environments," International Workshop of High Performance Computing for Computer Graphics and Visualization, Springer-Verlag, 1995, 217-237.

[12] I. Foster and N. Karonis, "A grid-enabled MPI: Message passing in heterogeneous distributed computing systems," Supercomputing'98, 1998.

[13] I. Foster and C. Kesselman, "Globus: A metacomputing infrastructure toolkit," International Journal of Supercomputer Applications, 11 (1997) 115-128. (Also at http: / / www.globus . org)

[14] I. Foster and C. Kesselman, The Grid: Blueprint for a New Computing Infrastructure, Morgan Kaufmann, 1999.

[15] A. Grimshaw, W. Wulf, and the Legion team, "The Legion vision of a worldwide virtual computer,' Communications of the ACM, 40 (1997) 3945.

[16] B. Hendrickson and R. Leland, "A multilevel algorithm for partitioning graphs," Technical Report SAND93-1301, Sandia National Laboratories, 1993

[17] G. Karypis and V. Kumar, "Parallel multilevel k-way partitioning scheme for irregular graphs," Technical Report 96-036, University of Minnesota, 1996.

[18] J. Leigh, A. Johnson, and T. DeFanti, "CAVERN: A distributed architecture for supporting scalable persistence and interoperability in collaborative virtual environments," Virtual Reality Research, Development and Applications 2 (1997) 217-237.

[19] M. Litzdow, M. Livny, and M.W. Mutka, "Condor - a hunter of idle workstations," 8th International Conference of Distributed Computing Systems, (1988) 104-111.

[20] S. Nog and D. Kotz, "A performance comparison of TCP/IP and MPI on FDDI, fast Ethernet, and Ethernet," Technical Report PCS-TR95-273, Dartmouth College, 1996.

[21] L. Oliker and R. Biswas, "PLUM: Parallel load balancing for adaptive unstructured meshes," Journal of Parallel and Distributed Computing, 52 (1998) 150-177.

[22] K. Schloegel, G. Karypis, and V. Kumar, "Multilevel diffusion schemes for repartitioning of adaptive meshes," Journal of Parallel and Distributed Computing, 47 (1997) 109-124.

[23] C. Walshaw, M. Cross, and M. Everett, "Parallel dynamic graph partitioning for adaptive unstructured meshes," Journal of Parallel and Distributed Computing, 47 (1997), 102-108. 
TABLE I

SCALABILITY ANALYSIS OF THE TEST APPLICATION

\begin{tabular}{|c|c|c|c|c|c|c|c|c|c|c|c|}
\hline & \multicolumn{11}{|c|}{ Number of Processors } \\
\hline Latency & 2 & 4 & 8 & 16 & 32 & 64 & 128 & 256 & 512 & 1024 & 2048 \\
\hline Max. Tolerance & 3777 & 1824 & 1148 & 614 & 324 & 168 & 89 & 72 & 58 & 51 & 57 \\
\hline No Tolerance & 4547 & 3193 & 1699 & 1033 & 558 & 302 & 173 & 123 & 115 & 109 & 103 \\
\hline
\end{tabular}

TABLE II

EXPECTED RUNTIME FOR VARYING ThrOTTle VALUES

\begin{tabular}{|c|c|c|c|c|c|c|c|c|c|c|}
\multicolumn{2}{c|}{} & \multicolumn{10}{|c|}{ ThroTTle values } \\
\hline Metric & Clusters & 0 & 1 & 3 & 4 & 16 & 32 & 64 & 128 & $200 \mathrm{k}$ \\
\hline \hline MaxQWgt & 1 & 1993 & 1427 & 348 & 312 & 291 & 300 & 306 & 312 & 324 \\
& 2 & 1847 & 1142 & 748 & 467 & 320 & 304 & 305 & 318 & 345 \\
& 3 & 2035 & 1801 & 674 & 556 & 375 & 331 & 324 & 326 & 382 \\
& 4 & 1868 & 1516 & 761 & 639 & 412 & 352 & 328 & 371 & 425 \\
& 5 & 1834 & 1626 & 835 & 767 & 438 & 373 & 359 & 343 & 400 \\
& 6 & 2081 & 1579 & 898 & 825 & 481 & 391 & 357 & 361 & 427 \\
& 7 & 1884 & 1279 & 1032 & 758 & 505 & 383 & 371 & 369 & 414 \\
& 8 & 1944 & 1451 & 1102 & 834 & 531 & 434 & 376 & 380 & 435 \\
\hline LoadImb & 1 & 7.05 & 5.09 & 1.23 & 1.11 & 1.01 & 1.00 & 1.00 & 1.00 & 1.00 \\
& 2 & 8.54 & 4.16 & 2.74 & 1.81 & 1.26 & 1.14 & 1.04 & 1.00 & 1.00 \\
& 3 & 7.15 & 6.40 & 2.50 & 2.11 & 1.41 & 1.19 & 1.05 & 1.02 & 1.01 \\
& 4 & 6.63 & 5.41 & 2.82 & 2.40 & 1.58 & 1.26 & 1.07 & 1.03 & 1.01 \\
& 5 & 6.53 & 5.78 & 3.06 & 2.83 & 1.66 & 1.30 & 1.11 & 1.02 & 1.01 \\
& 6 & 7.31 & 5.58 & 3.25 & 2.99 & 1.81 & 1.40 & 1.08 & 1.02 & 1.01 \\
& 7 & 6.68 & 4.61 & 3.74 & 2.80 & 1.84 & 1.33 & 1.10 & 1.03 & 1.00 \\
& 8 & 6.90 & 5.15 & 3.92 & 3.05 & 1.94 & 1.43 & 1.13 & 1.06 & 1.00 \\
\hline
\end{tabular}

TABLE III

EXPECTED RUNTIME IN THOUSANDS OF UNITS FOR VARYING CLUSTERS AND INTERCONNECT SPEEDS $(P=32)$

\begin{tabular}{||c|c|r|r|r||}
\cline { 2 - 5 } \multicolumn{1}{c|}{} & \multicolumn{4}{c|}{ Interconnect Speeds } \\
\hline Clusters & 3 & 10 & \multicolumn{1}{c|}{100} & \multicolumn{1}{c|}{1000} \\
\hline 1 & 473 & 473 & 473 & 473 \\
2 & 728 & 863 & 1228 & 4102 \\
3 & 755 & 1168 & 2783 & 18512 \\
4 & 791 & 1361 & 3667 & 25040 \\
5 & 854 & 1649 & 5677 & 53912 \\
6 & 915 & 1717 & 8521 & 76169 \\
7 & 956 & 1915 & 10958 & 80568 \\
8 & 968 & 2178 & 11492 & 93566 \\
\hline
\end{tabular}

(a) No latency tolerance

\begin{tabular}{||c|r|r|r|r||}
\cline { 2 - 5 } \multicolumn{1}{c|}{} & \multicolumn{4}{c|}{ Interconnect Speeds } \\
\hline Clusters & 3 & 10 & \multicolumn{1}{c|}{100} & \multicolumn{1}{c|}{1000} \\
\hline 1 & 287 & 287 & 287 & 287 \\
2 & 298 & 469 & 763 & 3941 \\
3 & 322 & 548 & 2386 & 12705 \\
4 & 328 & 680 & 3297 & 21888 \\
5 & 336 & 768 & 4369 & 33092 \\
6 & 345 & 856 & 5044 & 52668 \\
7 & 352 & 893 & 5480 & 61079 \\
8 & 357 & 1048 & 5721 & 61321 \\
\hline
\end{tabular}

(b) Maximum latency tolerance 